International Journal of Pure and Applied Mathematics

Volume 83 No. 3 2013, 465-475

ISSN: 1311-8080 (printed version); ISSN: 1314-3395 (on-line version)

url: http://www.ijpam.eu

doi: http://dx.doi.org/10.12732/ijpam.v83i3.7

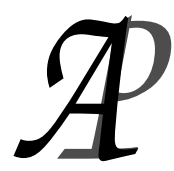

ijpam.eu

\title{
FOUR KINDS OF DIFFERENTIABLE MAPS
}

\author{
Cheng'en $\mathrm{Yu}^{1} \S$, Xiaolan $\mathrm{Liu}^{2}$ \\ ${ }^{1,2}$ School of Science \\ Sichuan University of Science and Engineering \\ Zigong, Sichuan, 643000, P.R. CHINA
}

\begin{abstract}
The B-differentiability of $f$ is defined in general vector normed spaces, which is defined in finite dimensional Euclidean space(See [2]). Meanwhile, Gäteaux differentiability, Hadamard differentiability and Fréchet differentiability are also considered. In addition, srong Fréchet differentiability and strong Bouligand differentiability are also introduced. We present the relationships along these kinds of differentiable maps and give two examples to explain these relationships.
\end{abstract}

AMS Subject Classification: 90C25, 90C33

Key Words: Gäteaux differentiable; Hadamard differentiable; Fréchet differentiable; Bouligand-differentiable; Strong B-derivative; Strong F-derivative.

\section{Introduction}

For later discussion, some definitions and lemmas are introduced. Let $X$ and

Received: October 30, 2012

(c) 2013 Academic Publications, Ltd. url: www.acadpubl.eu

${ }^{\S}$ Correspondence author 
$Y$ be vector normed spaces and consider a mapping $f: X \rightarrow Y$.

Definition 1.1. (see [1]) A mapping $f: X \rightarrow Y$ is said to be directionally differentiable at a point $x$ along a direction $h$ if the limit

$$
f^{\prime}(x ; h):=\lim _{t \downarrow 0} \frac{f(x+t h)-f(x)}{t}
$$

exists; this limit, denoted $f^{\prime}(x ; h)$, is called the directional derivative of $f$. If $f$ is directionally differentiable at $x$ in every direction $h \in X$, we say that $f$ is directionally differentiable at $x$.

Remark 1.1. The directional derivative $f^{\prime}(x ; h)$, when it exists, is positively homogeneous in $h$, i.e., $f^{\prime}(x ; t h)=t f^{\prime}(x ; h)$ for any $t \geq 0$.

Definition 1.2. (see [1]) A mapping $f: X \rightarrow Y$ is said to be Gäteaux differentiable at a point $x$ if $f$ is directionally differentiable at a point $x$ and the directional derivative $f^{\prime}(x ; h)$ is linear and continuous in $h$. That is, $f^{\prime}(x ; h)$ : $X \rightarrow Y$ is a linear continuous operator. We denote this operator(when it exists) by $f^{\prime}(x)$, i.e., $f^{\prime}(x ; h)=f^{\prime}(x) h$.

Definition 1.3. (see [1]) A mapping $f: X \rightarrow Y$ is said to be directionally differentiable at a point $x$ in the Hadamard sense if the directional derivative $f^{\prime}(x ; h)$ exists for all $h$ and, moreover,

$$
f^{\prime}(x ; h):=\lim _{\substack{t, 0 \\ h^{\prime} \rightarrow h}} \frac{f\left(x+t h^{\prime}\right)-f(x)}{t} .
$$

If in addition $f^{\prime}(x ; h)$ is linear in $h, f$ is Hadamard differentiable at $x$. Equation (2) can be formulated in the following equivalent form, for any sequences $h_{n} \rightarrow h$ and $t_{n} \downarrow 0$ :

$$
f^{\prime}(x ; h):=\lim _{n \rightarrow \infty} \frac{f\left(x+t_{n} h_{n}\right)-f(x)}{t_{n}} .
$$

Before Bouligand differentiable, we introduce the concept of Lipschitz continuity. 
Definition 1.4. A mapping $f: X \rightarrow Y$ is said to be locally Lipschitz continuous at $x$ if there exists a constant $L>0$ such that

$$
\|f(y)-f(x)\| \leq L\|y-x\|, \quad \forall y \in \mathbb{N}(x),
$$

where $\mathbb{N}(x)$ denotes the neighborhood of $x$.

It is said that $f$ is Lipschitz continuous on a set $S \subset X$ if there exists a constant $L>0$ such that

$$
\left\|f\left(x_{1}\right)-f\left(x_{2}\right)\right\| \leq L\left\|x_{1}-x_{2}\right\|, \quad \forall x_{1}, x_{2} \in S .
$$

Definition 1.5. A mapping $f: X \rightarrow Y$ is said to be Bouligand differentiable(for short, B-differentiable) at a point $x$ if $f$ is Lipschitz continuous in a neighborhood of $x$ and directionally differentiable at $x$. If $f$ is B-differentiable at $x$, we call the directional derivative $f^{\prime}(x ; h)$ the B-derivative of $f$ at $x$ along $h$.

The B-derivative $f^{\prime}(x ; h)$ is strong if the error function

$$
e(y)=f(y)-f(x)-f^{\prime}(x ; y-x)
$$

satisfies

$$
\lim _{\substack{y^{1} \neq y^{2} \\\left(y^{1}, y^{2}\right) \rightarrow(x, x)}} \frac{e\left(y^{1}\right)-e\left(y^{2}\right)}{\left\|y^{1}-y^{2}\right\|}=0 .
$$

In this case, we say that $f$ is strongly B-differentiable at $x$. We say that $f$ is B-differentiable near $x$ if $f$ is B-differentiable at every point in a certain neighborhood of $x$.

Remark 1.2. Definition 1.5 is extended from B-differentiability of functional $f$ in $[2]$ to general operator, i.e., $\mathbb{R}^{n}$ is replaced by general vector normed space $X, \mathbb{R}^{m}$ is replaced by general vector normed space $Y$, that is, finite dimensional Euclidean space is extended to infinite dimensional space.

Definition 1.6. (see [1]) A mapping $f: X \rightarrow Y$ is said to be directionally differentiable at $x$ in the Fréchet sense if $f$ is directionally differentiable at $x$ and

$$
\lim _{h \downarrow 0} \frac{f(x+h)-f(x)}{\|h\|}=f^{\prime}(x ; h)
$$


In other words, we can write (8) as follows:

$$
f(x+h)=f(x)+f^{\prime}(x ; h)+o(\|h\|), \quad h \in X .
$$

If in addition, $f^{\prime}(x ; h)$ is linear and continuous, it is said that $f$ is Fréchet differentiable(for short, F-differentiable) at $\mathrm{x}$. If $f$ is F-differentiable at $x$, we call the directional derivative $f^{\prime}(x ; h)$ is the F-derivative of $f$ at $x$ along $h$.

The F-derivative is strong at $x$ if

$$
\lim _{\substack{y^{1} \neq y^{2} \\\left(y^{1}, y^{2}\right) \rightarrow(x, x)}} \frac{f\left(y^{1}\right)-f\left(y^{2}\right)-f^{\prime}(x)\left(y^{1}-y^{2}\right)}{\left\|y^{1}-y^{2}\right\|}=0 .
$$

\section{Relationship Along Kinds of Differentiable Maps}

We all know that the Fréchet differentiability implies Gäteaux differentiability, for continuously differentiable mappings, Fréchet differentiability is equivalent to Gäteaux differentiability. If the space $X$ is finite dimensional, then Hadamard directionally differentiable implies Fréchet directionally differentiable. If $f$ is Fréchet directionally differentiable and $f^{\prime}(x ; \cdot)$ is continuous, then the converse is also true, i.e., Hadamard directionally differentiability holds. In addition, if $X$ is finite dimensional and $f$ is locally Lipschitz continuous, then the Fréchet directionally differentiability, Hadamard directionally differentiability and Bouligand differentiability are equivalent.

Give some results relevant to kinds of differentiability. The following proposition shows that the relationship between Fréchet differentiability and Bouligand differentiability, including the relationship between strong Fréchet differentiability and strong Bouligand differentiability.

Proposition 2.1. (i)If $f: X \rightarrow Y$ is locally Lipschitz continuous and has an $F$-derivative at $x$, then $f$ is B-differentiable at $x$.

(ii)If $f$ has a strong $F$-derivative at $x$, then $f$ has a strong B-derivative at $x$. 
Proof. (i): Since $f$ has an F-derivative at $x$, then $f$ is directionally differentiable at $x$. By the assumption, $f$ is locally Lipschitz continuous at $x$, then $f$ is B-differentiable at $x$.

(ii): Since $f$ has a strong $\mathrm{F}$-derivative at $x$, i.e,

$$
\lim _{\substack{y^{1} \neq y^{2} \\\left(y^{1}, y^{2}\right) \rightarrow(x, x)}} \frac{f\left(y^{1}\right)-f\left(y^{2}\right)-f^{\prime}(x)\left(y^{1}-y^{2}\right)}{\left\|y^{1}-y^{2}\right\|}=0 .
$$

Since

$$
e\left(y^{1}\right)=f\left(y^{1}\right)-f(x)-f^{\prime}(x)\left(y^{1}-x\right),
$$

and

$$
e\left(y^{2}\right)=f\left(y^{2}\right)-f(x)-f^{\prime}(x)\left(y^{2}-x\right),
$$

then it follows from (11), (12) and (13) that

$$
\begin{aligned}
& \lim _{\substack{y^{1} \neq y^{2} \\
\left(y^{1}, y^{2}\right) \rightarrow(x, x)}} \frac{e\left(y^{1}\right)-e\left(y^{2}\right)}{\left\|y^{1}-y^{2}\right\|} \\
= & \lim _{\substack{y^{1} \neq y^{2} \\
\left(y^{1}, y^{2}\right) \rightarrow(x, x)}} \frac{f\left(y^{1}\right)-f(x)-f^{\prime}(x)\left(y^{1}-x\right)-\left[f\left(y^{2}\right)-f(x)-f^{\prime}(x)\left(y^{2}-x\right)\right]}{\left\|y^{1}-y^{2}\right\|} \\
= & \lim _{\substack{y^{1} \neq y^{2} \\
\left(y^{1}, y^{2}\right) \rightarrow(x, x)}} \frac{f\left(y^{1}\right)-f\left(y^{2}\right)-f^{\prime}(x)\left(y^{1}-y^{2}\right)}{\left\|y^{1}-y^{2}\right\|} \\
= & 0 .
\end{aligned}
$$

So, $f$ has a strong B-derivative at $x$.

The following proposition presents that if $f$ is B-differentiable at $x$, globally Lipschitz continuity of B-derivative $f^{\prime}(x ; \cdot)$ and the equivalence conditions of strong B-derivative $f^{\prime}(x ; \cdot)$ are obtained.

Proposition 2.2. If $f$ is $B$-differentiable at $x$, then

(i) B-derivative $f^{\prime}(x ; \cdot)$ is a globally Lipschitz continuous function with respect to second variable and $f$ is directionally differentiable at $x$ in the Hadamard sense. 
(ii)B-derivative $f^{\prime}(x ; \cdot)$ is strong if and only if the error function $e$ has a strong $F$-derivative at $x$ and $F$-derivative of $e$ is equal to 0.

Proof. (i): By the definition of B-derivative $f^{\prime}(x ; \cdot)$, we have that

$$
\begin{aligned}
\left\|f^{\prime}\left(x ; h_{1}\right)-f^{\prime}\left(x ; h_{2}\right)\right\| & =\left\|\lim _{t \downarrow 0} \frac{f\left(x+t h_{1}\right)-f(x)}{t}-\lim _{t \downarrow 0} \frac{f\left(x+t h_{2}\right)-f(x)}{t}\right\| \\
& =\left\|\lim _{t \downarrow 0} \frac{f\left(x+t h_{1}\right)-f\left(x+t h_{2}\right)}{t}\right\| \\
& \leq \lim _{t \downarrow 0} \frac{\left\|f\left(x+t h_{1}\right)-f\left(x+t h_{2}\right)\right\|}{t} \\
& \leq \lim _{t \downarrow 0} L \frac{\left\|t h_{1}-t h_{2}\right\|}{t} \\
& =L\left\|h_{1}-h_{2}\right\|, \quad \forall h_{1}, h_{2} \in X
\end{aligned}
$$

where $L$ is the Lipschitz constant of $f$. Thus, B-derivative $f^{\prime}(x ; \cdot)$ is a globally Lipschitz continuous function with respect to second variable.

On the other hand, we have that

$$
\frac{f\left(x+t h^{\prime}\right)-f(x)}{t}=\frac{f(x+t h)-f(x)}{t}+\frac{f\left(x+t h^{\prime}\right)-f(x+t h)}{t},
$$

and by the Lipschitz continuity of $f$,

$$
\left\|\frac{f\left(x+t h^{\prime}\right)-f(x+t h)}{t}\right\| \leq L\left\|h^{\prime}-h\right\| .
$$

As $t \downarrow 0, \frac{f\left(x+t h^{\prime}\right)-f(x)}{t} \rightarrow \frac{f(x+t h)-f(x)}{t}$, then (2) follows from (1).

(ii)" $\Rightarrow$ ": first we calculate the $e^{\prime}(x ; h)$ :

$$
\begin{aligned}
e^{\prime}(x ; h) & =\lim _{t \downarrow 0} \frac{e(x+t h)-e(x)}{t} \\
& =\lim _{t \downarrow 0} \frac{f(x+t h)-f(x)-f^{\prime}(x ; t h)-\left[f(x)-f(x)-f^{\prime}(x ; x-x)\right]}{t} \\
& =0 .
\end{aligned}
$$

Since B-derivative $f^{\prime}(x ; \cdot)$ is strong, that is

$$
\lim _{\substack{y^{1} \neq y^{2} \\\left(y^{1}, y^{2}\right) \rightarrow(x, x)}} \frac{e\left(y^{1}\right)-e\left(y^{2}\right)}{\left\|y^{1}-y^{2}\right\|}=0 .
$$


Then it follows from the above two that

$$
\lim _{\substack{y^{1} \neq y^{2} \\\left(y^{1}, y^{2}\right) \rightarrow(x, x)}} \frac{e\left(y^{1}\right)-e\left(y^{2}\right)-e^{\prime}(x ; h)\left(y^{1}-y^{2}\right)}{\left\|y^{1}-y^{2}\right\|}=0 .
$$

$" \Leftarrow "$ : The error function $e$ has a strong $\mathrm{F}$-derivative at $x$, that is

$$
\lim _{\substack{y^{1} \neq y^{2} \\\left(y^{1}, y^{2}\right) \rightarrow(x, x)}} \frac{e\left(y^{1}\right)-e\left(y^{2}\right)-e^{\prime}(x ; h)\left(y^{1}-y^{2}\right)}{\left\|y^{1}-y^{2}\right\|}=0 .
$$

Since by the calculation of $e^{\prime}(x ; h), e^{\prime}(x ; h)=0$. Thus, we have that

$$
\lim _{\substack{y^{1} \neq y^{2} \\\left(y^{1}, y^{2}\right) \rightarrow(x, x)}} \frac{e\left(y^{1}\right)-e\left(y^{2}\right)}{\left\|y^{1}-y^{2}\right\|}=0 .
$$

So B-derivative $f^{\prime}(x ; \cdot)$ is strong.

Remark 2.1. It follows from the proof of (ii) in Proposition 2.2 that if $f$ is directionally differentiable at $x$, then $e^{\prime}(x ; h)=0$, i.e, $e^{\prime}(x)=0$ if $f$ is F-differentiable at $x$.

If $f$ is $\mathrm{B}$-differentiable in a neighborhood of $x$, the B-derivative $f^{\prime}(\cdot ; h)$ is generally not continuous with respect to first viable. But if for every fixed direction $h, f^{\prime}(\cdot ; h)$ is continuous with respect to first viable at $x$, then $f$ is F-differentiable at $x$. The following proposition say that: if $f$ is directionally differentiable at $x$ in the Hadamard sense, then the directional derivative $f^{\prime}(x ; \cdot)$ is continuous on $X$.

Theorem 2.1. If $f$ is directionally differentiable at $x$ in the Hadamard sense, then the directional derivative $f^{\prime}(x ; \cdot)$ is continuous on $X$.

Proof. Assume that $f$ is directionally differentiable at $x$ in the Hadamard sense and consider two directions $h, h^{\prime} \in X$. Without loss of generality we can assume that $f(x)=0$. Then for $\forall \epsilon>0$ and sufficiently small $t>0$, we have that

$$
\left\|f^{\prime}\left(x ; h^{\prime}\right)-\frac{f\left(x+t h^{\prime}\right)-f(x)}{t}\right\|=\left\|f^{\prime}\left(x ; h^{\prime}\right)-\frac{f\left(x+t h^{\prime}\right)}{t}\right\| \leq \epsilon .
$$


Moreover, by the definition of directionally differentiability at $x$ in the Hadamard sense, we also have that, for $h^{\prime}$ sufficiently close to $h$ and sufficiently small $t>0$,

$$
\left\|f^{\prime}(x ; h)-\frac{f\left(x+t h^{\prime}\right)}{t}\right\| \leq \epsilon .
$$

It follows that

$$
\left\|f^{\prime}(x ; h)-f^{\prime}\left(x ; h^{\prime}\right)\right\| \leq 2 \epsilon .
$$

Since $\epsilon$ is arbitrary, this proof is completed.

The composition of two B-differentiable functions has some interesting properties. First, it remains B-differentiable; there is a chain rule. Moreover, it is possible to establish that the B-derivative of the composite map is strong under an appropriate restriction.

Theorem 2.2. Let $f: X \rightarrow Y$ and $g: Y \rightarrow Z$ be B-differentiable at $x \in X$ and $f(x) \in Y$ respectively. Suppose that $f(X) \subseteq Y$. The following two statements hold.

(i)The composite map $\psi \equiv g \circ f: X \rightarrow Z$ is B-differentiable at $x \in X$; moreover,

$$
\psi^{\prime}(x ; h)=g^{\prime}\left(f(x) ; f^{\prime}(x ; h)\right), \quad \forall h \in \mathbb{R}^{n}
$$

(ii)If $g$ is strongly $F$-differentiable at $f(x)$ and $f$ has a strong $B$-derivative at $x$, then $\psi$ has a strong $B$-derivative at $x$.

Proof. We prove (ii) only; the following proof is applicable to (i) with a minor modification. It suffices to show

$$
\lim _{\substack{1 \\ y^{1} \neq y^{2} \\\left(y^{1}, y^{2}\right) \rightarrow(x, x)}} \frac{e_{\psi}\left(y^{1}\right)-e_{\psi}\left(y^{2}\right)}{\left\|y^{1}-y^{2}\right\|}=0 .
$$

where

$$
\begin{gathered}
e_{\psi}(y) \equiv g(f(y))-g(f(x))-g^{\prime}(f(x) ; f(y)-f(x)) \\
\equiv g(f(y))-g(f(x))-g^{\prime}\left(f(x) ; f^{\prime}(x ; y-x)\right),
\end{gathered}
$$


where Equation 22 holds since $f$ has a strong B-derivative at $x$.

Since $g$ is F-differentiable at $v \equiv f(x)$, thus $g^{\prime}(v ; \cdot)$ is linear in the second variable, we have, for $i=1,2$,

$$
e_{\psi}\left(y^{i}\right)=e_{g}\left(f\left(y^{i}\right)\right)+g^{\prime}(f(x)) e_{f}\left(y^{i}\right),
$$

where

$$
e_{g}(u) \equiv g(u)-g(v)-g^{\prime}(v)(u-v), \quad \forall u \in \mathbb{R}^{m},
$$

and

$$
e_{f}(y) \equiv f(y)-f(x)-f^{\prime}(x)(y-x), \quad \forall y \in \mathbb{R}^{m},
$$

Since $g$ has a strong F-derivative at $v$, then $g$ has a strong B-derivative at $v$, we have

$$
\lim _{\substack{u^{1} \neq u^{2} \\\left(u^{1}, u^{2}\right) \rightarrow(v, v)}} \frac{e_{g}\left(u^{1}\right)-e_{g}\left(u^{2}\right)}{\left\|u^{1}-u^{2}\right\|}=0 .
$$

Hence

$$
\begin{aligned}
& \lim _{\substack{y^{1} \neq y^{2} \\
\left(y^{1}, y^{2}\right) \rightarrow(x, x)}} \frac{e_{g}\left(f\left(y^{1}\right)\right)-e_{g}\left(f\left(y^{2}\right)\right)}{\left\|y^{1}-y^{2}\right\|} \\
= & \lim _{\substack{y^{1} \neq y^{2} \\
\left(y^{1}, y^{2}\right) \rightarrow(x, x)}} \frac{e_{g}\left(f\left(y^{1}\right)\right)-e_{g}\left(f\left(y^{2}\right)\right)}{\| f\left(y^{1}-f\left(y^{2}\right) \|\right.} \cdot \frac{\| f\left(y^{1}-f\left(y^{2}\right) \|\right.}{\left\|y^{1}-y^{2}\right\|} \\
= & 0,
\end{aligned}
$$

where the last equality holds because $f$ is locally Lipschitz continuous at $x$. Similarly, since $f$ has a B-derivative at $x$, we have

$$
\lim _{\substack{y^{1} \neq y^{2} \\\left(y^{1}, y^{2}\right) \rightarrow(x, x)}} \frac{e_{f}\left(y^{1}\right)-e_{f}\left(y^{2}\right)}{\left\|y^{1}-y^{2}\right\|}=0 .
$$

Combining the last two expressions immediately yields the desired limit (21).

By the Part(i) of Proposition 2.2, If $f$ is B-differentiable at $x$, then $f$ is directionally differentiable at $x$ in the Hadamard sense. Then combing Theorem 2.2 with Proposition 2.2, the following corollary is obtained. 
Corollary 2.1. If $f$ is directionally differentiable at $x$ and that $g$ is Hadamard directionally differentiable at $v \equiv f(x)$. Then the composite mapping $g \circ f$ is directionally differentiable at $x$ and the following chain rule holds:

$$
(g \circ f)^{\prime}(x, h)=g^{\prime}\left(f(x) ; f^{\prime}(x, h)\right)
$$

Remark 2.2. (i) It is important to note that in part (b) of the above Theorem 2.2, the order of composition is important; more precisely, if $f$ has a strong F-derivative at $x$ and $g$ has a strong B-derivative at $f(x)$, the composition $g \circ f$ does not necessarily have a strong B-derivative at $x$.

(ii)Corollary 2.1 is Proposition 2.47 in [1]. The order of composition is also important; if $f$ is Hadamard directionally differentiable at $x$ and that $g$ is directionally differentiable at $v \equiv f(x)$, then the composite mapping $g \circ f$ is directionally differentiable at $x$. Moreover, it is not difficult to see that if in the above Corollary 2.1 the mapping $f$ is also Hadamard directionally differentiable at $x$, then the composite mapping is Hadamard directionally differentiable as well.

The following two examples show that relationship along these kinds of differentiable maps.

Example 1. (see [1]) Consider the Hilbert space $X:=L_{2}[0,1]$, the set $K \subset L_{2}[0,1]$ formed by almost everywhere nonnegative valued functions $x(\cdot) \in$ $L_{2}[0,1]$, and the function $f(x):=d(x, K): X \rightarrow \mathbb{R}$. The function $f(x)$ is a continuous and convex function, since the set $K$ is convex. It is difficult to see that $f(x)=\left\|x_{-}\right\|$, where $x_{-}(t):=\min \{x(t), 0\}, t \in[0,1]$. Consider the function $x_{0}(t)=1$ for all $t \in[0,1]$. Clearly, $x_{0} \in K$, and it is not difficult to check that $f$ is Gäteaux differentiable at $x_{0}$, with Gäteaux derivative $f^{\prime}(x ; h)=0$, i.e., $f^{\prime}(x)=0$. On the other hand, consider the sequence of functions $h_{n}(t):=$ $-\left(1+2 \alpha_{n}\right) t^{\alpha_{n}}, t \in[0,1]$, where $\alpha_{n}$ is a sequence of numbers such that $\alpha_{n} \downarrow-\frac{1}{2}$. Note that $\left\|h_{n}\right\|=\left(1+2 \alpha_{n}\right)^{\frac{1}{2}} \rightarrow 0$. By direct calculations it is possible to verify that $f\left(x_{0}+h_{n}\right) /\left\|h_{n}\right\| \rightarrow e^{-1} \neq 0$. Consequently, we obtain that the function $f$ is not Fréchet differentiable at $x_{0}$. 
The example shows that it can happen that a convex continuous function is Gäteaux differentiable and Hadamard differentiable, but not Fréchet differentiable.

Example 2. (see [2]) Let

$$
f\left(r, x_{1}, x_{2}\right):=\min \left(\frac{r_{+}}{\sqrt{x_{1}^{2}+x_{2}^{2}}}, 1\right) x, \quad\left(r, x_{1}, x_{2}\right) \in \mathbb{R}^{3}
$$

We claim that this function is B-differentiable everywhere on $\mathbb{R}^{3}$. First we show that $f$ is globally Lipschitz continuous on $\mathbb{R}^{3}$. Second, we can prove that $f$ directionally differentiable everywhere on $\mathbb{R}^{3}$.

\section{Acknowledgments}

This work is partially supported by the Scientific Research Fund of Sichuan Provincial Education Department (12ZA098), the Scientific Research Fund of Sichuan University of Science and Engineering (2012KY08), the Scientific Research Fund of School of Science SUSE (10LXYB03).

\section{References}

[1] J.F. Bonnans, A. Shapiard, Perturbation Analysis of Optimazation Problems, Springer-Verlag, New York (2000).

[2] F. Facchinei, J.-S. Pang, Finite-Dimensional Variational Inequalities and Complementarity Problems, Springer-Verlag, New York (2003). 
